\title{
Paraneoplastic Pemphigus With Cicatricial Nail Involvement
}

\author{
Brian J. Simmons, MD; Jennifer A. Adams, MD; Jeong Hee Cho-Vega, MD, PhD; Antonella Tosti, MD
}

\section{PRACTICE POINTS}

- Paraneoplastic pemphigus (PNP) is a rare blistering skin eruption commonly associated with an underlying malignancy.

- Paraneoplastic pemphigus generally presents with erosive stomatitis with involvement of the vermillion lip but also can involve the skin and nails.

- Nail involvement can lead to scarring of the nails and can mimic lichen planus, bullous pemphigoid, or epidermolysis bullosa of the nails. These nail changes likely are due to the pronounced lichenoid interphase dermatitis seen in PNP.

To the Editor:

Paraneoplastic pemphigus (PNP), also known as paraneoplastic autoimmune multiorgan syndrome, is an autoimmune mucocutaneous blistering disease that typically occurs secondary to a lymphoproliferative disorder. Paraneoplastic pemphigus is characterized by severe erosive stomatitis, polymorphous skin lesions, and potential bronchiolitis obliterans that can mimic a wide array of conditions. The exact pathogenesis is unknown but is thought to be due to a combination of humoral and cellmediated immunity. The condition usually confers a poor prognosis, with morbidity from $38 \%$ to upwards of $90 \%{ }^{1}$

A 47-year-old man developed prominent pink to dusky, ill-defined, targetoid, coalescing papules over the back; violaceous macules over the palms and soles; and numerous crusted oral erosions while hospitalized for an infection. He had a history of stage IVB follicular lymphoma (double-hit type immunoglobulin heavy chain/ BCL2 fusion and rearrangement of BCL6) complicated by extensive erosive skin lesions and multiple lines of infections. The clinical differential diagnosis included StevensJohnson syndrome vs erythema multiforme (EM) major secondary to administration of oxacillin vs PNP. Herpes simplex virus polymerase chain reaction and Mycoplasma titers were negative. Skin biopsies from the back and right abdomen revealed severe lichenoid interface dermatitis (IFD) with numerous dyskeratotic cells mimicking EM and eosinophils; however, direct immunofluorescence of the abdomen biopsy revealed an apparent suprabasal acantholysis with intercellular C3 in the lower half of the epidermis. Histologically, PNP was favored, but indirect immunofluorescence with monkey esophagus IgG was negative.

The skin lesions progressed, and an additional skin biopsy from the left arm performed 1 month later revealed similar histologic features with intercellular IgG and $\mathrm{C} 3$ in the lower half of the epidermis with weak basement membrane C3 (Figure 1). Serology also confirmed elevated serum antidesmoglein 1 and 3 antibodies. Thus, in the clinical setting of an erosive mucositis with EM-like and pemphigoidlike eruptions associated with B-cell lymphoma, the patient was diagnosed with PNP.

Despite multiple complications followed by intermittent treatments, the initial therapy with rituximab

Dr. Simmons is from the Department of Dermatology, Dartmouth-Hitchcock Medical Center, Lebanon, New Hampshire. Dr. Adams is from the Department of Dermatology, University of Nebraska Medical Center, Omaha. Drs. Cho-Vega and Tosti are from the University of Miami Miller School of Medicine, Florida. Dr. Tosti is from the Department of Dermatology and Cutaneous Surgery, and Dr. Cho-Vega is from the Department of Pathology, Dermatopathology Division.

The authors report no conflict of interest.

Correspondence: Brian J. Simmons, MD, Dartmouth-Hitchcock Medical Center, Department of Dermatology, One Medical Center Dr, Lebanon, NH 03756 (brian.j.simmons@hitchcock.org).

doi:10.12788/cutis.0098 
induction and subsequent cycles of R-CHOP (rituximab, cyclophosphamide, doxorubicin hydrochloride, vincristine sulfate, and prednisone) for the B-cell lymphoma was done during his hospital stay. Toward the end of his 8-week hospitalization, the patient was noted to have
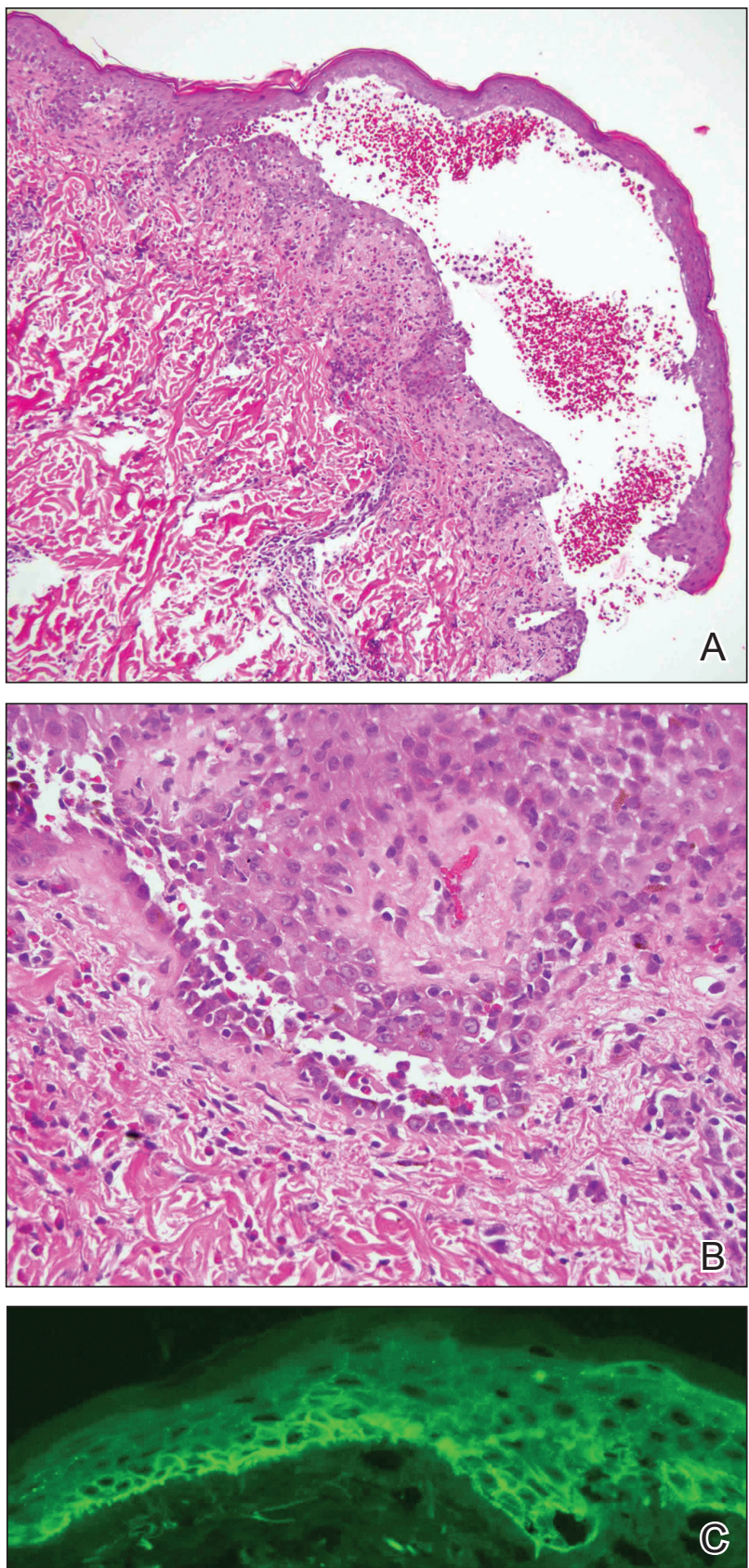

FIGURE 1. A, Histologic sections of an abdominal skin biopsy specimen showed an intraepidermal bulla with acantholysis and numerous eosinophils in the epidermis ( $\mathrm{H} \& \mathrm{E}$, original magnification $\times 20)$. $B$, Apparent suprabasal acantholysis and numerous dyskeratotic cells were best shown at high-power view (H\&E, original magnification $\times 400)$. C, Direct immunofluorescence revealed intercellular IgG and C3 deposits in the lower epidermis with a weak basement membrane deposit (original magnification $\times 200$ ). new lesions involving the hands, digits, and nails. The left hand showed anonychia of several fingers with prominent scarring (Figure 2A). There were large, verrucous, crusted plaques on the distal phalanges of several fingers on the right hand (Figure 2B). At that time, he was taking $20 \mathrm{mg}$ daily of prednisone (for 10 months) and had completed his 6th cycle of R-CHOP, which resulted in improvement of the skin lesions. Oral steroids were tapered, and he was maintained on rituximab infusions every 8 weeks but has since been lost to follow-up.

Paraneoplastic lymphoma is a rare condition that affects $0.012 \%$ of non-Hodgkin lymphoma and chronic lymphocytic leukemia patients. ${ }^{2}$ Reports of PNP involving
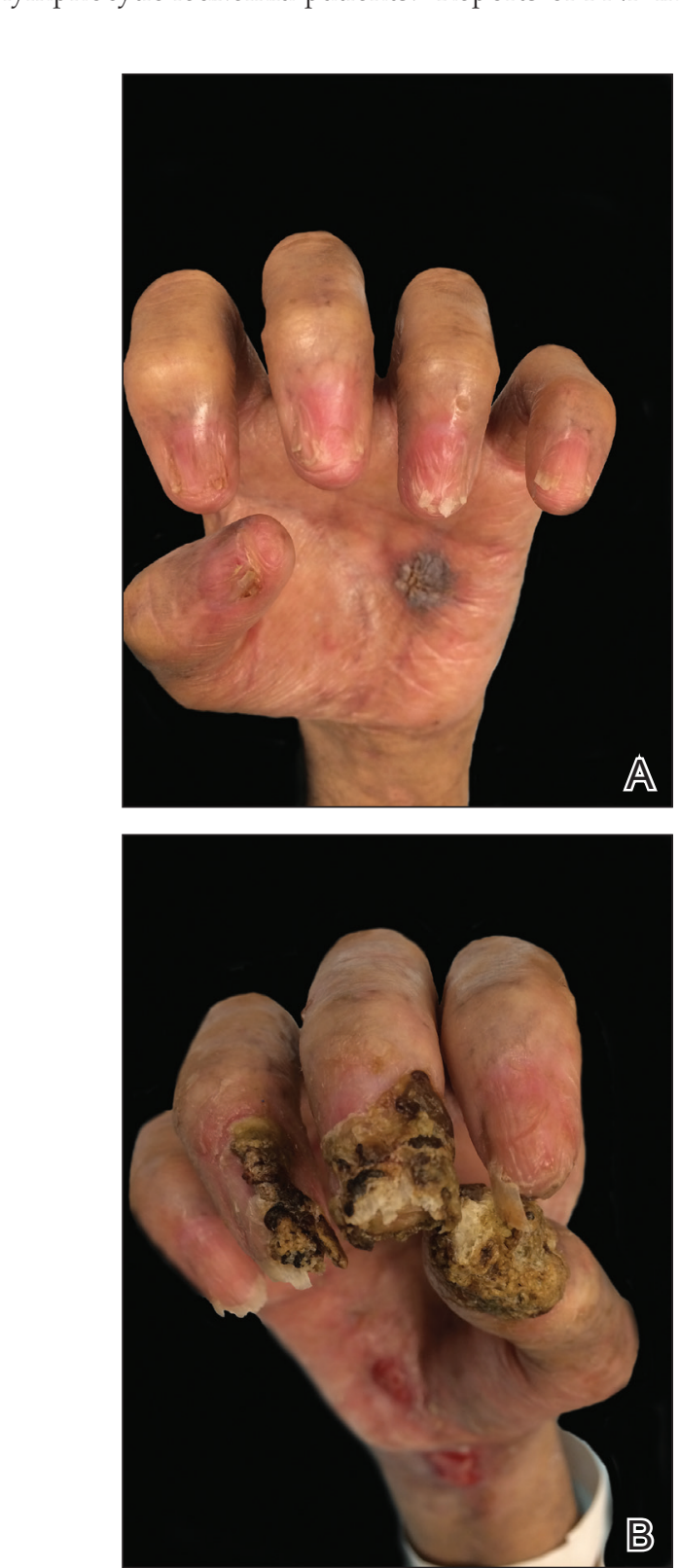

FIGURE 2. A, Anonychia of multiple fingers was seen on the left hand with nail bed scarring and paronychia. B, Exophytic crust was observed on multiple fingernails on the right hand. 
the nails are even more rare, with 3 reports in the setting of underlying Castleman disease $\mathrm{e}^{3-5}$ and 2 reports in patients with underlying non-Hodgkin ${ }^{6}$ and follicular ${ }^{1}$ lymphoma. These studies describe variable nail findings ranging from periungual erosions and edema, formation of dorsal pterygium, onycholysis with longitudinal furrowing, and destruction of the nail plate leading to onychomadesis and/or anonychia. These nail changes typically are seen in lichen planus or in bullous diseases affecting the basement membrane (eg, bullous pemphigoid, acquired epidermolysis bullosa) but not known in pemphigus, which is characterized by nonscarring nail changes. ${ }^{7}$

Although antidesmoglein 3 antibody was shown to be a pathologic driver in PNP, there is a weak correlation between antibody profiles and clinical presentation. ${ }^{8}$ In one case of PNP, antidesmoglein 3 antibody was negative, suggesting that lichenoid IFD may cause the phenotypic findings in PNP. ${ }^{9}$ Thus, the development of nail scarring in PNP may be explained by the presence of lichenoid IFD that is characteristic of PNP. However, the variation in antibody profile in PNP likely is a consequence of epitope spreading.

\section{REFERENCES}

1. Miest RY, Wetter DA, Drage LA, et al. A mucocutaneous eruption. Int J Dermatol. 2014;53:1425-1427.

2. Anhalt GJ, Mimouni D. Paraneoplastic pemphigus. In: LA G, Katz SI, Gilchrest, eds. Fitzpatrick's Dermatology in General Medicine 8th Edition. Vol 1. New York, NY: McGraw Hill; 2012:600.

3. Chorzelski T, Hashimoto T, Maciejewska B, et al. Paraneoplastic pemphigus associated with Castleman tumor, myasthenia gravis and bronchiolitis obliterans. J Am Acad Dermatol. 1999;41:393-400.

4. Lemon MA, Weston WL, Huff JC. Childhood paraneoplastic pemphigus associated with Castleman's tumour. $\mathrm{Br} J$ Dermatol. 1997;136:115-117.

5. Tey HL, Tang MB. A case of paraneoplastic pemphigus associated with Castleman's disease presenting as erosive lichen planus. Clin Exp Dermatol. 2009;34:e754-e756.

6. Liang JJ, Cordes SF, Witzig TE. More than skin-deep. Cleve Clin J Med. 2013;80:632-633.

7. Tosti A, Andre M, Murrell DF. Nail involvement in autoimmune bullous disorders. Dermatol Clin. 2011;29:511-513, xi.

8. Ohyama M, Amagai M, Hashimoto T, et al. Clinical phenotype and anti-desmoglein autoantibody profile in paraneoplastic pemphigus. J Am Acad Dermatol. 2001;44:593-598.

9. Kanwar AJ, Vinay K, Varma S, et al. Anti-desmoglein antibody-negative paraneoplastic pemphigus successfully treated with rituximab. Int $J$ Dermatol. 2015;54:576-579. 